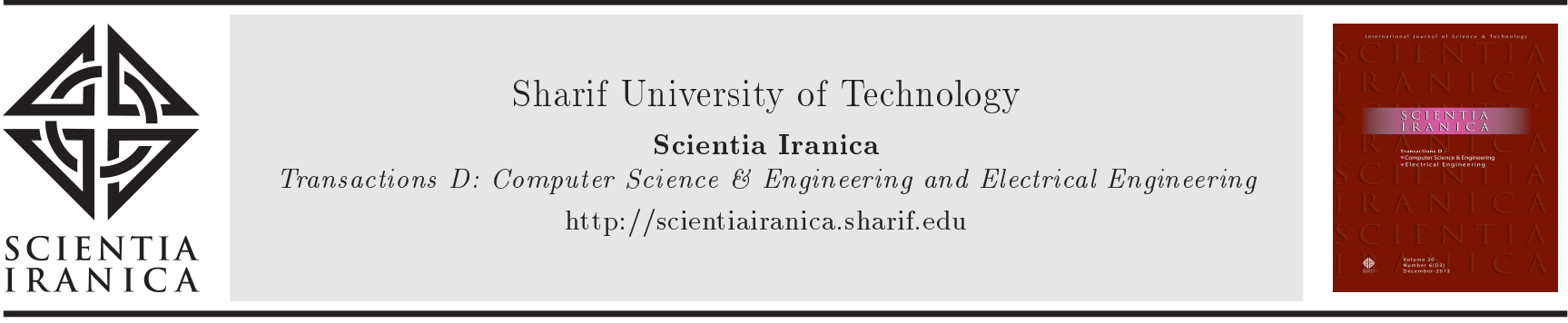

Research Note

\title{
Extending concepts of mapping of human brain to artificial intelligence and neural networks
}

\author{
A. Joghataie* \\ Department of Civil Engineering, Structural Engineering Group, Sharif University of Technology, Tehran, Iran.
}

Received 23 June 2019; received in revised form 29 December 2019; accepted 20 May 2020

\author{
KEYWORDS \\ Neural networks; \\ Artificial intelligence; \\ AIculus; \\ NNculus; \\ Autonomous robots; \\ Brain mapping; \\ Cortical homunculus; \\ Sensory homunculus.
}

\begin{abstract}
This paper introduces the concept of mapping of Artificially Intelligent (AI) computational systems. The concept of homunculus from human neurophysiology is extended to AI systems. It is assumed that an AI system behaves similarly to a mini-column or ganglion in the natural animal brain that comprises a layer of afferent (input) neurons, a number of interconnecting processing cells, and a layer of efferent (output) neurons or organs. The objective of the present study was to identify the correlation between the stimulus to each afferent neuron and the corresponding response from each efferent organ when the intelligent system is subjected to certain stimuli. To clarify the general concept, a small three-layered feedforward Neural Network (NN) was used as a simple example and an NNculus was built. Two important applications of this concept lie in the quality control of autonomous robots where an NN or AI culi can be built to evaluate their performance and in investigation of the topographic organization in the internal layers of the mini-columns of the human brain through hardware or numerical simulations using artificial NN.
\end{abstract}

(C) 2021 Sharif University of Technology. All rights reserved.

\section{Introduction}

The rapid progress in producing autonomous robots, which are expected to be capable of decision making and autonomous functioning, is bringing new concepts and issues that are gradually becoming more challenging. While building autonomous robots has always been an advanced complicated technological problem; nowadays, the main focus is shifted to their applications.

A number of researchers in all fields of science and technology are now trying to set some universally accepted rules and regulations to keep the building and operation of autonomous robots within some universal limits before the mass production of autonomous

\footnotetext{
*. E-mail address: joghatae@sharif.ir
}

robots. Mapping the artificial brains of autonomous robots is a useful hardware-based approach to their quality control and investigation of whether suitable associations have occurred in their autonomous responsebehavior system. Though it might seem that the available and already-made robots are not yet at that level of advancement and sophistication that make their manufacturers map the information processing systems of their products, the technology of making intelligent and highly autonomous robots is not farfetched.

\subsection{Three main issues}

The issues related to autonomous robots fall within three main categories: the moral requirements that should be practiced by the designers of autonomous robots that have been recently studied by many authors and organizations [1]; moral guidelines that should be implemented within the autonomous robots to guide 
them how to make and apply appropriate decisions according to some specific human criteria [2]; and rights of autonomous robots which, as a challenging issue, have not been the focus of study yet.

A fundamental question still remains in all of the above issues, i.e., how to consider the criteria and guidelines in production and usage of autonomous robots. The significance of the above mentioned issues as well as the expected immense impact of the presence of autonomous robots on all aspects of human life made the United Nations form a commission and group work to set definitions and a fuzzy frame work for production and usage of autonomous robots [3]. Some authors such as Anderson and Anderson [4] have emphasized that the industries were required to draw special attention to the ethical issues in production of autonomous robots. The operation-related issues of autonomous robots have been the focus of a number of recent debates and discussions [5]. An important application of autonomous robots lies in elderly health care where assistive robots are designed to provide help and support to the elderly. This issue has gained significance in recent years due to the increasing number of aging populations of many industrial countries. Japan and Italy, for example, are two countries with a small percentage of young population, implying that in the near future, the younger generation maybe outnumbered by the older and thus, intelligent robots and devices will be employed to help those elderly people who are in need of physical and mental assistance. In fact, artificial devices and robots function as complementary assistants to the older generation [6].

\subsection{AIculus and quality control}

Building what is called in this paper an AI-culus for an Artificially Intelligent (AI) system is a helpful step in its quality control. This process can be applied to autonomous robots. By developing and comparing the AI-culi of different brands of autonomous robots, their capabilities, advantages, and disadvantages can be compared. The AI-culus is a characteristic of the robot. One of the main objectives of this paper was to introduce this concept and show how an AI-culus could be built for a simple intelligent computational system. The example AI system, employed in this paper, is a multi-layer feedforward Neural Network (NN). Allocation of information in an AI system, for example in multi-layer $\mathrm{NN}$, gains significance in terms of size optimization of the intelligent system. Mapping of the intelligent system can reveal how the information is kept in the $\mathrm{NN}$ and whether or not this allocation of information is properly done.

\subsection{Deep learning, AI and artificial NN}

Information processing module of any autonomous robot uses artificial NN in one or another way. Ar- tificial NN have been extensively used in modeling the brain for both neuroscience studies and industrial applications. A very common and widely used type of $\mathrm{NN}$ is the multilayer $\mathrm{NN}$ with a layered structure similar to the minicolumns of the brain. In fact, artificial NN are considered as models of the hardware of natural brain. Brain minicolumns seem to play a key role in many brain characteristics and functions. Most importantly, some researchers have found evidences revealing the strong correlation between brain diseases and anomalies of the minicolumns $[7,8]$.

\subsection{Research methodology used in this study}

Basically, this research is a numerical investigation by computer modelling of the problem. The idea and method for natural brain mapping was adopted and applied to the NN. An extensive investigation of autonomous and sensory homunculi was performed and the findings were employed as the background and supporting references for this research.

\section{Homunculus and Al-culus}

An advanced form of autonomous robots would use some forms of artificial brain, which would probably be a hybrid system made of both artificial and biological organisms. However, the next generations of autonomous robots will probably use an advanced artificial brain which may be a combination of three types of processing systems: the traditional AI, simulated NN, and NN hardware. The artificial brain hardware should be tested to ensure it functions satisfactorily. Another alternative would be to build a map of the artificial brain similar to that of the human brain cortex used to study localization in the human brain. The concept of human brain mapping was first suggested by Penfield and Jasper about 70 years ago [9]. He applied a stimulus signal to different points of the cortex of a patient's brain and studied the response of the motor system of the patient to the input. By examining an array of points on the cortex, Penfield built a map of the cortex and tried to identify which point on the cortex was connected to which limb and organ of the body such as the hands, feet, eyes, and ears. Penfield considered the portion of the cortex area allocated to each limb or organ as a measure to assess the importance of that organ to the brain. He even drew a disproportioned shape of the human body, as viewed by the brain cortex, called "homunculus". Whether or not a sensory and cortical homunculus represent the real distribution of functionality within the brain is still an interesting subject for further research $[10,11]$.

Cortex mapping and building a homunculus were both novel ideas because they provided a hardwarebased approach to the study of human behavior. The objective of mapping was to better understand the 
topographic organization of the brain, given the high localization observed in the animal brain $[12,13]$. The new research points to the existence of large assemblies of networks within the brain [14]. On the experimental side, there have been developments on recording animal brain activities both at cellular and NN levels [15].

An important question may arise here, i.e., whether and how this localization occurs in an artificial brain. The extension of concepts of the natural brain mapping to the artificial brains of autonomous machines can play a significant role in the quality control, assessment of functioning, and safety of autonomous systems.

\subsection{AIculus and studying the human brain}

While mapping the natural brain is mainly performed to identify the outermost layer of the brain neurons connected directly to the motor neurons of a limb or organ, mapping of an artificial brain can be done for a larger area of the artificial brain, not only for its last layer that is directly connected to output organs. The structure and organization of the central and peripheral nervous system have been well studied and identified [16]. Moyes and Schulte [11] noted that mapping the internal layers of human brain was a useful idea to better understand the brain behavior and functioning: an objective that remains too hard to achieve so far. Further, investigation of the association areas in animals would be of little use for drawing any conclusion about the human brain since the association areas in the frontal lobe of the human brain is 6 times of chimpanzees' that have probably the most similar animal brains to the human brain [11]. Hence an accurate human brain simulation is of great significance to study and build a map for the internal layers of the brain. The model can then be used in building an AIculus which can approximately represent the homunculus.

\subsection{Receptor inputs and building AIculus}

An AIculus is built for the inputs to which the artificial intelligence is expected to be subjected. In most practical cases, especially for an autonomous robot, the robot designer should define and identify the domain space of the stimuli that the robot is expected to experience during its operation. Obviously, it is a large volume of data in most cases.

\section{A simple example problem of building a NN-culus}

In this section, the idea of building an AIculus is applied to the small multilayer feedforward NN shown in Figure 1. NN have been used as adaptive learning systems in many scientific and engineering applications for many years. A number of recent applications are [17] in engineering and [18] medicine. The NN

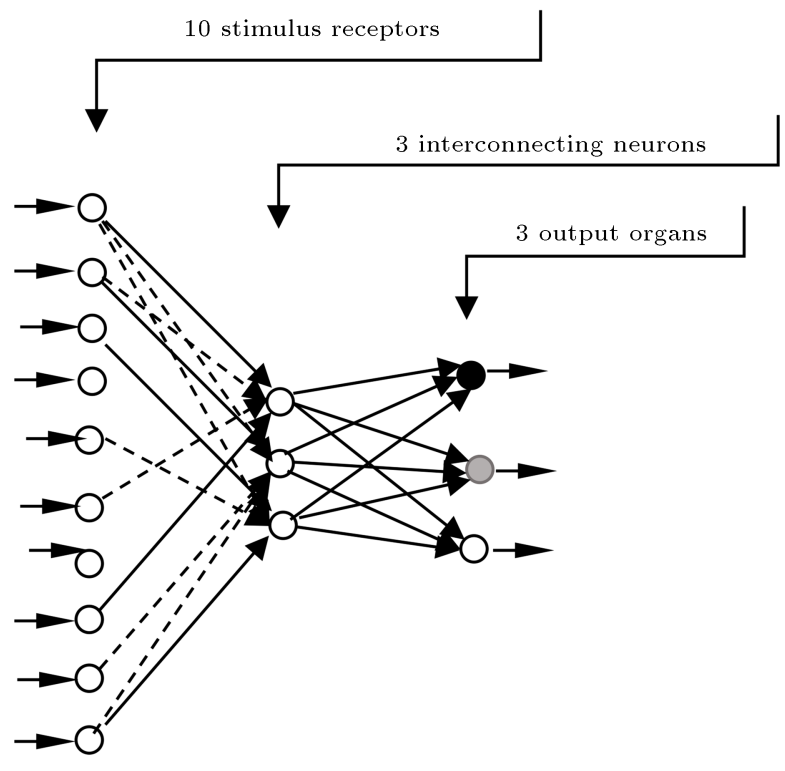

Figure 1. The ganglion multi-layer feed-forward neural network of this study. The left layer is the input layer with 10 input receptors. There are 3 interconnecting neurons in the middle layer. There are 3 output organs distinguished by their colors.

considered in this paper represents a ganglion with 3 layers:

1. An input layer with 10 input receptors;

2. An output layer with 3 response organs;

3. An interconnecting layer which embeds 3 integrating neurons.

For better visualization of the results, each output is shown by a different color. Organ 1 is shown in black, organ 2 in grey, and organ 3 in white. Each interconnecting neuron makes a dendritic synapse with each of the input receptors and has an axon with three distal branches that connects the interconnecting neuron to the output organs. Each receptor neuron transfers the stimulus input it receives according to the simple equation $y=x$ where $x$ is the stimulus input intensity and $y$ is the receptor's action potential. The interconnecting neurons as well as the output organs issue their action potentials based on the sigmoidal function $y=2 /\left(1+e^{-c z}\right)-1$, where $c$ is the characteristic constant of the neuron or organ, $z$ is the weighted sum of all its inputs, and $y$ is the action potential issued by the neuron or organ. This type of sigmoidal neuron has been widely used by many researchers including the author of this article $[19,20]$.

In this example, $c=0.1$. The synaptic weights of the NN have been randomly selected. It was assumed that NN will be subjected to 12 input vectors during its operation. The 12 stimuli input vectors were generated 
from the following arbitrarily defined equation:

$$
X_{j}^{i}=2 \sin [\pi(j+i-12) / 24],
$$

which represents the stimulus to receptor number $j=$ $1,2, \cdots, 10$, when input vector number $i=1,2, \cdots, 12$ was applied. Each input vector was fed to the NN and the response at the layer of organs of the NN was determined and recorded.

\subsection{Sensitivity of output organs responses to stimuli}

The response sensitivity of each organ to the stimulus applied to each receptor cell corresponding to a given stimulus vector was determined by sensitivity analysis. To numerically determine the sensitivity, a minor change was applied to the stimulus value and any change in each organ's output was determined. The ratio of the change in the organ's output to the change in stimulus was calculated as the sensitivity. Mathematically, the sensitivities can be expressed as:

$$
S_{k, j}^{i}=\Delta y_{k}^{i} / \Delta x_{j}^{i},
$$

where $k=1,2,3$ is the organ number and $y_{k}^{i}$ is the output response of organ $k$ due to input vector number $i$.

\subsection{Using sensitivities to determine the correlations between receptors and output organs}

To determine which receptor is most correlated with which output organ, different statistical models can be applied. In this example, a simple approach was used based on the calculated sensitivities. The correlation problem can be stated as follows: consider the input receptor number $j$ and determine which output organ is most sensitive to the inputs of this receptor.

To find a solution for the above problem, a simple algorithm was used in this paper. The output organ was determined based on the average of the absolute values for all sensitivities. Consider receptor number $j$ and organ number $k$. The average sensitivity of organ $k$ to receptor $j$ was determined by:

$$
S_{k, j}^{\prime}=\sum_{i=1}^{12}\left|S_{k, j}^{i}\right| / 12
$$

where $|\ldots|$ stands for the absolute value for (...). For receptor number $j, S_{k, j}^{\prime}$ was calculated for each of the three output organs, $k=1,2,3$, and the organ with the largest $S^{\prime}$ was determined as the most sensitive organ to receptor $j$. This procedure was repeated for all the receptors and the organ most sensitive to each receptor was identified. The results of this simple problem are shown in Figure 2 where each receptor is given the color of its corresponding most sensitive organ.

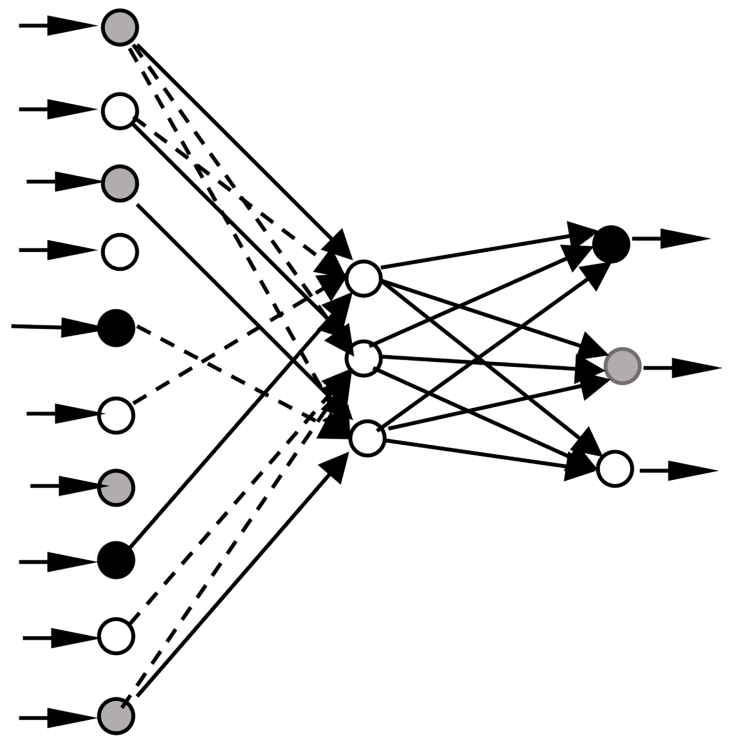

Figure 2. The Neural Network (NN) of Figure 1 showing the result of mapping. The receptor cells are colored to identify with which output organ they are correlated. Each receptor is given the color of its most correlated output organ.

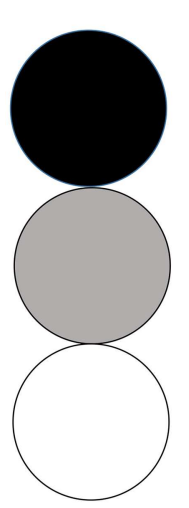

(a)

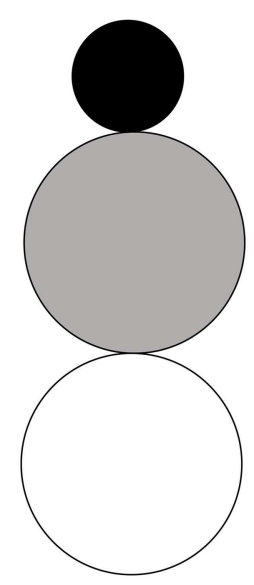

(b)
Figure 3. Comparing the real output organs with NNculus of the example problem: (a) Output organs in reality, (b) The NNculus which is a disproportioned shape of the real output organs.

\subsection{Building the NNculus of this example}

As deduced from Figure 2, mapping of the output organs to the receptors array (similar to cortex neurons) would yield an unequal distribution. There are two black, four grey, and four white receptors. The NNculus represents the significance of each output organ as viewed by the receptors array (cortex). Figure 3 shows the NNculus where the diameters of the circles represent the significance of the organs. The diameter of the black organ which is sensitive to two receptors, is one half the diameters of the white and gray organs, each of which are most sensitive to four receptors. 


\section{Application of AI and NN-culi in performance assessment of autonomous robots}

Building an AI-culus or NN-culus for a robot can provide very useful information about the performance of the robot. Consider two autonomous robots that are trained based on the same data but with different training paths and random parameters affecting their learning. Autonomous robots receive information from an array of sensors which is similar to the human sensory system. Moreover, they act in different ways similar to how the humans act. Therefore, mapping of sensory inputs on the artificial brain of a robot might be used to assess how the information is allocated in the brain and the relative importance of the sensory inputs with respect to each other. The same explanation can be made about the projection of the AI processors to the motor organs of the robot. A graphical demonstration of this assessment is the AI-culus. In the homunculus, the hands and tongue are much larger than the other body organs because these organs play more vital roles in the human life than the rest of the body. Whether this explanation is correct or not is not of the concern in this paper; however, the important point is that we might use a similar approach to assess the properties of brain-body relationship in autonomous robots. For example, in case the manufacturer of a robot expects input type A to be the main data in determining the activities of the robot while data type $\mathrm{B}$ occupies a larger area in the brain and, consequently, a larger part in the AI-culus, the robot can be ill-trained. The manufacturer may decide to change the training strategy to improve this situation. However, finding any relationship between the proportions of an NN-culus or AI-culus of a robot and its behaviour requires a detailed study of the subject and it is not possible to draw any general and reliable conclusion about this matter in this paper.

\section{Conclusion}

The concept of Homunculus representing the relative importance of body organs as viewed by the human brain cortex was extended to artificial intelligence and neural networks. A simple neural network was employed to demonstrate how the concept could be applied. An NNculus was built for the neural network. An AIculus could be also built for any Artificially Intelligent (AI) system. The concept can be applied to any type of intelligent system.

\section{References}

1. Lin, P., Abney, K., and Bekey, G.A., Robot Ethics. MIT Press, Cambridge, MA, USA (2014).
2. Wallach, W. and Allen, C., Moral Machines: Teaching Robots Right From Wrong, Oxford University Press (2010).

3. COMEST. United Nation, World Commission on the Ethics of Scientific Knowledge and Technology, SHS/YES/COMEST-10/17/2 REV (2017).

4. Anderson, M. and Anderson, S.L. "Toward ensuring ethical behavior from autonomous systems: a case-supported principle-based paradigm", Industrial Robot: An International Journal, 42(4), pp. 324-331 Emerald Group Publishing Limited [ISSN 0143-991X], (2015). DOI: 10.1108/IR-12-2014-0434

5. Calo, R., Froomkin, A.M., and Kerr, A. (Editors), Robot Law., Edward Elgar Pub; First Edition (March $29,2016)$.

6. Van Wynsberghe, A., Healthcare Robots Ethics, Design and Implementation, Published 2016 by Routledge New York, NY 10017, USA (2016).

7. Casanova, M.F. "White matter volume increase and minicolumns in autism", Annals of Neurology, 56(3), p. 453 (2004).

8. Casanova, M.F. and Casanova, E.L. "The modular organization of the cerebral cortex: Evolutionary significance and possible links to neurodevelopmental conditions", Journal of Comparative Neurology, 07/2019, 527(10), pp. 1720-1730 (2019).

9. Penfield, W. and Jasper, H., Epilepsy and the Functional Anatomy of the Human Brain, Boston, Little, Brown \& Co (1954).

10. Morishita, T., Miki, K., and Inoue, T. "Penfield's homunculus and recent advances in brain mapping", World Neurosurgery (Elsevier), made available online, Nov. 27 (2019).

11. Gandhoke, G.S. et al. "Edwin boldrey and wilder penfield's homunculus: A life given by Mrs. Cantlie (in and out of realism", World Neurosurgery, 132, December 19, pp. 377-388 (2019).

12. Moyes, C.D. and Schulte, P.M., Principles of Animal Physiology, 3rd Ed. Pearson Education Inc (2016).

13. Finger, S., Minds Behind the Brain: A History of the Pioneers and Their Discoveries, Oxford University Press, USA (2000).

14. Di Plinoi, S. and Ebisch, S.J. "Brain network profiling defines functionally specialized cortical networks", Human Brain Mapping, 39(12), pp. 4689-4706 (2018).

15. Schroter, M. et al. "Mapping neuronal network dynamics in developing cerebral organoids", Frontiers in Cellular Neuroscience, 12, (Jan. 2018). DOI: 10.3389/conf.fncel.2018.38.00066

16. Krebs, C., Weinberg, J., Akesson, E., and Dilli, E., Lippincott Illustrated Reviews: Neuroscience, 2nd Ed., Wolters Kluwer (2017). 
17. Yaseen, Z.M., et al. "An enhanced extreme learning machine model for river flow forecasting: State-of-theart, practical applications in water resource engineering area and future research direction", Journal of Hydrology, 569, pp. 387-408 (2019).

18. Liu, X. et al. "Fine-tuning pre-trained convolutional neural networks for gastric precancerous disease classification on magnification narrow-band imaging images", Neurocomputing, Article in press (2019). https://doi.org/10.1016/j.neucom.2018.10.100

19. Joghataie, A. and Torghabehi, O.O. "Simulating dynamic plastic continuous neural networks by finite elements", IEEE Trans. Neural Netw. Learn. Syst, 25(8), pp. 1583-1587 (2014).

20. Joghataie, A., and Shafiei Dizaji, M. "Neuro-Skins: dynamics, plasticity and effect of neuron type and cell size on their response", Publisher: Springer US, Neural Process Letters, (2018).

https://doi.org/10.1007/s11063-018-9795-7

\section{Biography}

Abdolreza Joghataie received his PhD degree from the University of Illinois at Urbana-Champaign, USA, and joined Sharif University of Technology, Iran, in 1996. The author has been doing research and publishing articles in the area of intelligent systems including deep learning and neural networks. His recent book which is a story-type-report on the future of intelligent systems and autonomous robots, ADANculus: neuropsychology and mapping of a two-phase brain, is also related to the subject of this paper. 